\title{
ON INCREASING SOLUTIONS OF HALF-LINEAR DELAY DIFFERENTIAL EQUATIONS
}

\author{
SERENA MATUCCI AND PAVEL ŘEHÁK
}

\begin{abstract}
We establish conditions guaranteeing that all eventually positive increasing solutions of a half-linear delay differential equation are regularly varying and derive precise asymptotic formulae for them. The results presented here are new also in the linear case and some of the observations are original also for non-functional equations. A substantial difference is pointed out between the delayed and nondelayed case for eventually positive decreasing solutions.
\end{abstract}

\section{INTRODUCTION}

Let us consider the equation

$$
\left(r(t) \Phi\left(y^{\prime}(t)\right)\right)^{\prime}=p(t) \Phi(y(\tau(t)))
$$

where $r, p$ are positive continuous functions on $[a, \infty)$ and $\Phi(u)=|u|^{\alpha-1} \operatorname{sgn} u$ with $\alpha>1$. Throughout the paper, we assume (unless not stated otherwise) that $\tau$ satisfies the following conditions:

$$
\tau \in C^{1}, \tau^{\prime}>0, \tau(t) \leq t
$$

and

$$
\limsup _{t \rightarrow \infty} \frac{t}{\tau(t)}<\infty
$$

The above conditions imply $\lim _{t \rightarrow \infty} \tau(t)=\infty$, and are fulfilled by standard examples of delay, e.g., $\tau(t)=t-\sigma$ with $\sigma>0$, or $\tau(t)=\lambda t$ with $\lambda \in(0,1)$. Note that, in contrast to the linear case where an equation with a general delay can be transformed into an equation with a constant delay (see [15]), in the half-linear case, it makes a good sense to consider a general $\tau$. Solutions of (1.1) are understood in the classical sense, i.e., a solution $y$ of (1.1) is a $C^{1}$ function defined in an interval $I \subseteq[a, \infty)$ such that $r \Phi\left(y^{\prime}\right) \in C^{1}(I)$ and $y$ satisfies (1.1) in $I$.

Our aim is to describe asymptotic behavior of all eventually positive increasing solutions of (1.1) via the theory of regular variation. Regularly varying functions have been shown to be a very useful tool in studying asymptotic properties of various types of differential equations, see, in particular, the monograph

$M S C$ (2010): primary 34K25; secondary $26 \mathrm{~A} 12$.

Keywords: Half-linear differential equation, delayed differential equation, increasing solution, asymptotic behavior, regular variation.

The research of the second author has been supported by the grant GA20-11846S of the Czech Science Foundation. The first author has been partially supported by Gnampa, National Institute for Advanced Mathematics (INdAM). 
[14]. Linear and half-linear ordinary differential equations have been considered in the framework of regular variation, e.g., in $[2,3,5,7,8,17-19]$. Linear and half-linear functional differential equations have been studied in this framework in $[5,9-11,13,16,20,21]$. The typical result in the works $[9-11,13,20,21]$ is the existence of a regularly varying solution, obtained by means of a topological approach, as, for example, the Schauder-Tychonoff fixed point theorem. Our approach is different. We deal with the entire class of eventually positive increasing solutions (which we assume to be non empty), and we show that it is a subset of the class of regularly varying solutions. In addition, we derive a precise asymptotic formula for each solution. All our results are new also in the (functional) linear case, and some of them are new in the non-functional (half-linear as well as linear) case. A comparison with the existing results is discussed in remarks after the main results.

The paper is organized as follows. In the next section, we give some basic information on equation (1.1) and recall selected facts from the theory of regular variation, which will also include De Haan class $\Pi$. The main results are presented in Section 3. In addition to showing regular variation of increasing solutions and deriving asymptotic formulae, we briefly discuss also decreasing solutions; in particular, we show that under reasonable assumptions, they may exhibit quite a different behavior when compared with the solutions of non-delayed equations. Directions for possible future research are discussed as well. The last section contains all the proofs.

\section{Preliminaries}

As usual, the relation $f(t) \sim g(t)$ as $t \rightarrow \infty$ means $\lim _{t \rightarrow \infty} f(t) / g(t)=1$, the relation $f(t) \asymp g(t)$ as $t \rightarrow \infty$ means that $\exists c_{1}, c_{2} \in(0, \infty)$ such that $c_{1} g(t) \leq f(t) \leq$ $c_{2} g(t)$ for large $t$, and $f(t)=o(g(t))$ as $t \rightarrow \infty$ means that $\lim _{t \rightarrow \infty} f(t) / g(t)=0$.

As for nonoscillatory solutions of (1.1) (i.e., the solutions which are eventually of one sign), without loss of generality, we only work with positive solutions, i.e., with the class

$$
\mathcal{S}=\{y: y(t) \text { is a positive solution of (1.1) for large } t\} .
$$

We wish to include our results into the framework of a standard classification of nonoscillatory solutions, which is given in what follows. Because of the sign conditions on the coefficients, all positive solutions of (1.1) are eventually monotone. Therefore, any such solution belongs to one of the following disjoint classes:

$$
\mathcal{I S}=\left\{y \in \mathcal{S}: y^{\prime}(t)>0 \text { for large } t\right\}, \mathcal{D S}=\left\{y \in \mathcal{S}: y^{\prime}(t)<0 \text { for large } t\right\} .
$$

As for the nonemptiness of these classes as well as of the subclasses defined below, general conditions are not known, as far as we know. Partial results related to the problem of the existence of nonoscillatory solutions were obtained in [12]. Further, as a by-product of the investigation of half-linear delay differential equations within the framework of regular variation via a fixed point approach, some existence theorems under a setting which is close to ours can be found, e.g., in [11,20].

We shall focus on the class $\mathcal{I S}$. This class can be divided into the mutually disjoint subclasses:

$$
\mathcal{I S}_{\infty}=\left\{y \in \mathcal{I} \mathcal{S}: \lim _{t \rightarrow \infty} y(t)=\infty\right\}, \quad \mathcal{I S}_{B}=\left\{y \in \mathcal{I} \mathcal{S}: \lim _{t \rightarrow \infty} y(t)=b \in \mathbb{R}\right\}
$$


Define the so-called quasiderivative $y^{[1]}$ of $y \in \mathcal{S}$ by $y^{[1]}=r \Phi\left(y^{\prime}\right)$. We introduce the following convention

$$
\mathcal{I S}_{u, v}=\left\{y \in \mathcal{I S}: \lim _{t \rightarrow \infty} y(t)=u, \quad \lim _{t \rightarrow \infty} y^{[1]}(t)=v\right\}
$$

where $u, v \in\{B, \infty\}$. If $u=B[v=B]$, we mean that the value of the corresponding limit is a real nonzero number. Using this convention, we further distinguish the following types of solutions which form subclasses in $\mathcal{I} \mathcal{S}_{B}$ and $\mathcal{I S} \mathcal{S}_{\infty}$ (we only list those that are not a-priori excluded):

$$
\mathcal{I S}_{B, B}, \mathcal{I S}_{B, \infty}, \mathcal{I S}_{\infty, B}, \mathcal{I} \mathcal{S}_{\infty, \infty}
$$

In some places, we need to emphasize that the classes and subclasses of eventually positive increasing solutions are associated with a particular equation, say equation $(*)$; then we write

$$
\mathcal{I S}^{(*)}, \mathcal{I S}_{B}^{(*)}, \text { etc. }
$$

In the second part of this section, we recall basic information on the Karamata theory of regularly varying functions and the de Haan class $\Pi$; for more information see the monographs $[1,4,6]$.

A measurable function $f:[a, \infty) \rightarrow(0, \infty)$ is called regularly varying (at infinity) of index $\vartheta, \vartheta \in \mathbb{R}$, if

$$
\lim _{t \rightarrow \infty} f(\lambda t) / f(t)=\lambda^{\vartheta} \quad \text { for every } \lambda>0 ;
$$

we write $f \in \mathcal{R} \mathcal{V}(\vartheta)$. If $\vartheta=0$, then we speak about slowly varying functions; we write $f \in \mathcal{S} \mathcal{V}$, thus $\mathcal{S} \mathcal{V}=\mathcal{R} \mathcal{V}(0)$. Here, $\mathcal{R} \mathcal{V}$ stands either for "regularly varying" or $\mathcal{R} \mathcal{V}=\bigcup_{\vartheta \in \mathbb{R}} \mathcal{R} \mathcal{V}(\vartheta)$.

A function $f \in \mathcal{R} \mathcal{V}(\vartheta)$ if and only if there exists a function $L \in \mathcal{S} \mathcal{V}$ such that $f(t)=t^{\vartheta} L(t)$ for every $t$. The slowly varying component of $f \in \mathcal{R} \mathcal{V}(\vartheta)$ will be denoted by $L_{f}$, i.e., $L_{f}(t):=f(t) / t^{\vartheta}$.

The following result (the so-called Uniform Convergence Theorem, see, e.g., [1]) is one of the most fundamental theorems in the theory. Many important properties of $\mathcal{R} \mathcal{V}$ functions follow from it.

Proposition 2.1. If $f \in \mathcal{R} \mathcal{V}(\vartheta)$, then the relation (2.2) holds uniformly on each compact $\lambda$-set in $(0, \infty)$.

Another important result in the theory of $\mathcal{R} \mathcal{V}$ functions is the Representation Theorem (see, e.g., [1]).

Proposition 2.2. $f \in \mathcal{R} \mathcal{V}(\vartheta)$ if and only if

$$
f(t)=\varphi(t) t^{\vartheta} \exp \left\{\int_{t_{0}}^{t} \frac{\psi(s)}{s} \mathrm{~d} s\right\},
$$

$t \geq t_{0}$, for some $t_{0}>0$, where $\varphi, \psi$ are measurable with $\lim _{t \rightarrow \infty} \varphi(t)=C \in(0, \infty)$ and $\lim _{t \rightarrow \infty} \psi(t)=0$. A function $f \in \mathcal{R} \mathcal{V}(\vartheta)$ can alternatively be represented as

$$
f(t)=\varphi(t) \exp \left\{\int_{t_{0}}^{t} \frac{\omega(s)}{s} \mathrm{~d} s\right\}
$$


$t \geq t_{0}$, for some $t_{0}>0$, where $\varphi, \omega$ are measurable with $\lim _{t \rightarrow \infty} \varphi(t)=C \in(0, \infty)$ and $\lim _{t \rightarrow \infty} \omega(t)=\vartheta$.

A regularly varying function $f$ is said to be normalized regularly varying, and we write $f \in \mathcal{N} \mathcal{R} \mathcal{V}(\vartheta)$, if $\varphi(t) \equiv C$ in $(2.3)$ or in the alternative representation. If (2.3) holds with $\vartheta=0$ and $\varphi(t) \equiv C$, we say that $f$ is normalized slowly varying and we write $f \in \mathcal{N S} \mathcal{V}$. Clearly, if $f$ is a $C^{1}$ function and $\lim _{t \rightarrow \infty} t f^{\prime}(t) / f(t)=\vartheta$, then $f \in \mathcal{N} \mathcal{R} \mathcal{V}(\vartheta)$. Conversely, if $f \in \mathcal{N} \mathcal{R} \mathcal{V}(\vartheta) \cap C^{1}$, then $\lim _{t \rightarrow \infty} t f^{\prime}(t) / f(t)=\vartheta$.

The classes of regularly varying solutions of (1.1) are defined as follows:

$$
\begin{aligned}
& \mathcal{S}_{\mathcal{S} \mathcal{V}}=\mathcal{S} \cap \mathcal{S} \mathcal{V}, \quad \mathcal{S}_{\mathcal{R} \mathcal{V}}(\vartheta)=\mathcal{S} \cap \mathcal{R} \mathcal{V}(\vartheta), \\
& \mathcal{S}_{\mathcal{N} \mathcal{S} \mathcal{V}}=\mathcal{S} \cap \mathcal{N} \mathcal{S} \mathcal{V}, \quad \mathcal{S}_{\mathcal{N} \mathcal{R} \mathcal{V}}(\vartheta)=\mathcal{S} \cap \mathcal{N} \mathcal{R} \mathcal{V}(\vartheta) .
\end{aligned}
$$

The Karamata Integration Theorem (see, e.g., [1,4]) plays a very important role in our theory. Its statement can be summarized as follows.

Proposition 2.3. Let $L \in \mathcal{S} \mathcal{V}$.

(i) If $\vartheta<-1$, then $\int_{t}^{\infty} s^{\vartheta} L(s) \mathrm{d} s \sim t^{\vartheta+1} L(t) /(-\vartheta-1)$ as $t \rightarrow \infty$.

(ii) If $\vartheta>-1$, then $\int_{a}^{t} s^{\vartheta} L(s) \mathrm{d} s \sim t^{\vartheta+1} L(t) /(\vartheta+1)$ as $t \rightarrow \infty$.

(iii) If $\int_{a}^{\infty} L(s) / s \mathrm{~d} s$ converges, then $\widetilde{L}(t)=\int_{t}^{\infty} L(s) / s \mathrm{~d} s$ is a $\mathcal{S} \mathcal{V}$ function; if $\int_{a}^{\infty} L(s) / s \mathrm{~d} s$ diverges, then $\widetilde{L}(t)=\int_{a}^{t} L(s) / s \mathrm{~d} s$ is a $\mathcal{S} \mathcal{V}$ function; in both cases, $L(t) / \widetilde{L}(t) \rightarrow 0$ as $t \rightarrow \infty$.

Here are further useful properties of $\mathcal{R} \mathcal{V}$ functions. The proofs of (i)-(viii) are either easy or can be found in $[1,4]$. The proof of (ix) can be found in the last section.

\section{Proposition 2.4.}

(i) If $f \in \mathcal{R} \mathcal{V}(\vartheta)$, then $\ln f(t) / \ln t \rightarrow \vartheta$ as $t \rightarrow \infty$. It then clearly implies that $\lim _{t \rightarrow \infty} f(t)=0$ provided $\vartheta<0$, or $\lim _{t \rightarrow \infty} f(t)=\infty$ provided $\vartheta>0$.

(ii) If $f \in \mathcal{R} \mathcal{V}(\vartheta)$, then $f^{\alpha} \in \mathcal{R} \mathcal{V}(\alpha \vartheta)$ for every $\alpha \in \mathbb{R}$.

(iii) If $f_{i} \in \mathcal{R} \mathcal{V}\left(\vartheta_{i}\right), i=1,2, f_{2}(t) \rightarrow \infty$ as $t \rightarrow \infty$, then $f_{1} \circ f_{2} \in \mathcal{R} \mathcal{V}\left(\vartheta_{1} \vartheta_{2}\right)$.

(iv) If $f_{i} \in \mathcal{R} \mathcal{V}\left(\vartheta_{i}\right), i=1,2$, then $f_{1}+f_{2} \in \mathcal{R} \mathcal{V}\left(\max \left\{\vartheta_{1}, \vartheta_{2}\right\}\right)$.

(v) If $f_{i} \in \mathcal{R} \mathcal{V}\left(\vartheta_{i}\right), i=1,2$, then $f_{1} f_{2} \in \mathcal{R} \mathcal{V}\left(\vartheta_{1}+\vartheta_{2}\right)$.

(vi) If $f_{1}, \ldots, f_{n} \in \mathcal{R} \mathcal{V}, n \in \mathbb{N}$, and $R\left(x_{1}, \ldots, x_{n}\right)$ is a rational function with nonnegative coefficients, then $R\left(f_{1}, \ldots, f_{n}\right) \in \mathcal{R} \mathcal{V}$.

(vii) If $L \in \mathcal{S V}$ and $\vartheta>0$, then $t^{\vartheta} L(t) \rightarrow \infty, t^{-\vartheta} L(t) \rightarrow 0$ as $t \rightarrow \infty$.

(viii) If $f \in \mathcal{R} \mathcal{V}(\vartheta), \vartheta \neq 0$, then there exists $g \in C^{1}$ with $g(t) \sim f(t)$ as $t \rightarrow \infty$ and such that $\operatorname{tg}^{\prime}(t) / g(t) \rightarrow \vartheta$, whence $g \in \mathcal{N} \mathcal{R} \mathcal{V}(\vartheta)$. Moreover, $g$ can be taken such that $\left|g^{\prime}\right| \in \mathcal{N} \mathcal{R} \mathcal{V}(\vartheta-1)$.

(ix) If $\left|f^{\prime}\right| \in \mathcal{R} \mathcal{V}(\vartheta), \vartheta \in \mathbb{R}$, with $f^{\prime}$ being eventually of one sign and $f(t) \rightarrow 0$ or $f(t) \rightarrow \infty$ as $t \rightarrow \infty$, then $f \in \mathcal{N} \mathcal{R} \mathcal{V}(\vartheta+1)$.

Some other properties of $\mathcal{R} \mathcal{V}$ functions, needed in the proofs of the main results, are presented in some auxiliary lemmas in the last section.

Finally, we recall the definition of the De Haan class $\Pi$, together with some useful properties. 
A measurable function $f:[a, \infty) \rightarrow \mathbb{R}$ is said to belong to the class $\Pi$ if there exists a function $w:(0, \infty) \rightarrow(0, \infty)$ such that for $\lambda>0$

$$
\lim _{t \rightarrow \infty} \frac{(f(\lambda t)-f(t))}{w(t)}=\ln \lambda
$$

we write $f \in \Pi$ or $f \in \Pi(w)$. The function $w$ is called an auxiliary function for $f$. The class $\Pi$, after taking absolute values, forms a proper subclass of $\mathcal{S} \mathcal{V}$.

Next, we give some properties of the class $\Pi$. The proofs of (i)-(ii) can be found in the monographs $[4,6]$. For (iii), see, e.g., [17].

\section{Proposition 2.5.}

(i) If $f \in \Pi(v)$, then $v(t) \sim f(t)-\frac{1}{t} \int_{a}^{t} f(s) \mathrm{d} s$ as $t \rightarrow \infty$.

(ii) If $f \in \Pi$, then $\lim _{t \rightarrow \infty} f(t)=: f(\infty) \leq \infty$ exists. If the limit is infinite, then $f \in \mathcal{S} \mathcal{V}$. If the limit is finite, then $f(\infty)-f(t) \in \mathcal{S} \mathcal{V}$.

(iii) If $f^{\prime} \in \mathcal{R} \mathcal{V}(-1)$, then $f \in \Pi\left(t f^{\prime}(t)\right)$.

\section{Main Results}

Denote

$$
G(t)=\Phi^{-1}\left(\frac{t p(t)}{r(t)}\right)=\left(\frac{t p(t)}{r(t)}\right)^{\beta-1}
$$

where $\beta$ is the conjugate number of $\alpha$, i.e., $1 / \alpha+1 / \beta=1$. We start by establishing conditions guaranteeing slow variation of increasing solutions, for which we also derive asymptotic formulae.

Theorem 3.1. (I) Assume that $\int_{a}^{\infty} p(s) \mathrm{d} s=\infty$ and

$$
\lim _{t \rightarrow \infty} \frac{t^{\alpha-1}}{r(t)} \int_{a}^{t} p(s) \mathrm{d} s=0 .
$$

Then, $\mathcal{I S} \subset \mathcal{N S} \mathcal{V}$. If, in addition, $p \in \mathcal{R} \mathcal{V}(\delta)$ with $\delta>-1$, then for any $y \in \mathcal{I S}$, the following holds.

(i) If $\int_{a}^{\infty} G(s) \mathrm{d} s=\infty$, then $y$ satisfies the formula

$$
y(t)=\exp \left\{\int_{a}^{t}(1+o(1)) \frac{G(s)}{\Phi^{-1}(\delta+1)} \mathrm{d} s\right\}
$$

as $t \rightarrow \infty$. Moreover, $\mathcal{S}_{\mathcal{N S} \mathcal{V}}=\mathcal{S}_{\mathcal{S} \mathcal{V}}=\mathcal{I S}=\mathcal{I S}_{\infty, \infty}$.

(ii) If $\int_{a}^{\infty} G(s) \mathrm{d} s<\infty$, then $y$ satisfies the formula

$$
y(t)=N \exp \left\{-\int_{t}^{\infty}(1+o(1)) \frac{G(s)}{\Phi^{-1}(\delta+1)} \mathrm{d} s\right\}
$$

as $t \rightarrow \infty$, where $N:=\lim _{t \rightarrow \infty} y(t) \in(0, \infty)$. Moreover, $\mathcal{S}_{\mathcal{N} \mathcal{S} \mathcal{V}}=\mathcal{S}_{\mathcal{S} \mathcal{V}}=\mathcal{I S}=$ $\mathcal{I} \mathcal{S}_{B, \infty}$.

(II) Let the conditions in point (I) be satisfied and let $r \in \mathcal{R} \mathcal{V}(\gamma)$ hold. Then, $\gamma \geq \alpha+\delta$. For any $y \in \mathcal{I S}$, we have $y(t) \in \Pi\left(t y^{\prime}(t)\right)$ provided $\gamma=\alpha+\delta$. Moreover, if $\gamma=\alpha+\delta$ and $\int_{a}^{\infty} G(s) \mathrm{d} s<\infty$, or $\gamma>\alpha+\delta$, then

$$
N-y(t) \sim \frac{N}{\Phi^{-1}(\delta+1)} \int_{t}^{\infty} G(s) \mathrm{d} s \in \mathcal{R} \mathcal{V}((\delta+1-\gamma)(\beta-1)+1)
$$


as $t \rightarrow \infty$. In particular, if $\gamma=\alpha+\delta$, then $|N-y| \in \mathcal{S} \mathcal{V}$ and

$$
\frac{L_{p}^{\beta-1}(t)}{L_{r}^{\beta-1}(t)(N-y(t))}=o(1)
$$

as $t \rightarrow \infty$.

Condition (3.1) is necessary in a certain sense. More precisely, the following lemma holds.

Lemma 3.2. Let $r \in \mathcal{R} \mathcal{V}(\gamma)$ with $\gamma>\alpha-1$, and $\tau(t) \asymp t$ as $t \rightarrow \infty$. If there exists $y \in \mathcal{I S} \cap \mathcal{N S} \mathcal{V}$, then (3.1) holds. If, in addition, $p \in \mathcal{R} \mathcal{V}(\delta)$, then

$$
\lim _{t \rightarrow \infty} t^{\alpha+\delta-\gamma} \frac{L_{p}(t)}{L_{r}(t)}=0 .
$$

Note that if

$$
p \in \mathcal{R} \mathcal{V}(\delta), \quad r \in \mathcal{R} \mathcal{V}(\delta+\alpha)
$$

hold, then

$$
G(t)=\frac{1}{t}\left(\frac{L_{p}(t)}{L_{r}(t)}\right)^{\beta-1} \in \mathcal{R} \mathcal{V}(-1)
$$

Observe that (3.7) along with $\delta>-1$ yield $\gamma>\alpha-1$ (i.e., the assumption from Lemma 3.2). Further, condition (3.1) (as well as condition (3.6)) reduces to

$$
\lim _{t \rightarrow \infty} \frac{L_{p}(t)}{L_{r}(t)}=0
$$

which is (3.6) with $\gamma=\delta+\alpha$.

From the non-functional case (see [19]) we know that $\int_{a}^{\infty} r^{1-\beta}(s) \mathrm{d} s=\infty$ and

$$
\lim _{t \rightarrow \infty} \frac{t^{\alpha-1}}{r(t)} \int_{t}^{\infty} p(s) \mathrm{d} s=0
$$

imply $\mathcal{D S} \subset \mathcal{N S} \mathcal{V}$. Under the additional condition $p \in \mathcal{R} \mathcal{V}(\delta)$ with $\delta<-1$, asymptotic formulae similar to (3.2) and (3.3) can be established. In particular, $\mathcal{S V}$ solutions must be sought among decreasing solutions. This result can be understood as a certain complement to Theorem 3.1. However, as we will see, here we encounter quite a big difference between the functional and non-functional case. For instance, let $r(t)=1, \alpha=2$, and $\tau(t)=t-1$ in (1.1). Then $y(t)=$ $e^{-t^{2}}$ is a solution of (1.1) which takes here the form $y^{\prime \prime}(t)=p(t) y(t-1)$, where $p(t)=\left(4 t^{2}-2\right) e^{1-2 t}$. Clearly, $\lim _{t \rightarrow \infty} t^{2} p(t)=0$, and this condition implies also $\lim _{t \rightarrow \infty} t \int_{t}^{\infty} p(s) \mathrm{d} s=0$, i.e., condition (3.10). However, $y \in \mathcal{D S}$, but $y \notin \mathcal{R} \mathcal{V}$. Thus, we see that (in contrast to the situation for $\mathcal{I S}$ discussed in Theorem 3.1), for decreasing solutions in our framework, qualitative behavior may substantially change when passing from ordinary to functional equations. An open problem is whether or not $\int_{a}^{\infty} r^{1-\beta}(s) \mathrm{d} s=\infty$ and (3.10) imply $\mathcal{D S} \subset \mathcal{N} \mathcal{S} \mathcal{V}$ when assuming, in addition, that $p \in \mathcal{R} \mathcal{V}(\delta)$ with $\delta<-1$.

Note that if $p \in \mathcal{R} \mathcal{V}(\delta)$ with $\delta \neq-1$, then both (3.1) when $\delta>-1$, and (3.10) when $\delta<-1$, reduce to the condition

$$
\lim _{t \rightarrow \infty} \frac{t^{\alpha} p(t)}{r(t)}=0
$$


which can easily be seen from the Karamata theorem.

We proceed with a complementary case with respect to Theorem 3.1 in the sense that we study increasing solutions when $\delta<-1$ under the conditions (3.7) and (3.1) (which yield (3.9)). We shall prove regular variation of these solutions where the index is equal to

$$
\varrho:=\frac{-1-\delta}{\alpha-1},
$$

and derive asymptotic formulae. Denote

$$
H_{\tau}(t)=\left(t \tau^{\prime}(t)\right)^{\alpha-1} \frac{p(t)}{r(\tau(t))} .
$$

If (3.7) and the first condition in (3.12) hold, then $H_{\tau} \in \mathcal{R} \mathcal{V}(-1)$ and $H_{\tau}$ can be written as

$$
H_{\tau}(t)=\frac{1}{t}\left(\frac{t}{\tau(t)}\right)^{\delta+\alpha}\left(\tau^{\prime}(t)\right)^{\alpha-1} \frac{L_{p}(t)}{L_{r}(\tau(t))} .
$$

Since the convergence/divergence of the integrals $\int_{a}^{\infty} G$ and $\int_{a}^{\infty} H_{\tau}$ plays an important role, the following example is of interest.

Example 3.3. Assume for simplicity that $\tau^{\prime}(t) \sim \lambda$ with $\lambda \in(0,1]$ as $t \rightarrow \infty$; note that $\tau(t)=\lambda t$ or $\tau(t)=t-\sigma$ both satisfy this condition. Then,

$$
H_{\tau}(t) \sim \frac{\lambda^{-\delta-1} t^{\alpha-1} p(t)}{r(\tau(t))} \sim \frac{\lambda^{-\delta-1} t^{\alpha-1} p(t)}{r(t)}=\frac{\lambda^{-\delta-1}}{t} \cdot \frac{L_{p}(t)}{L_{r}(t)}
$$

as $t \rightarrow \infty$ by Lemma 4.1 provided (3.7) holds. Recall that $G$ takes here the form (3.8). Taking $r, p$ such that $L_{p}(t) / L_{r}(t) \sim \ln ^{\gamma} t$ as $t \rightarrow \infty, \gamma \in(-\infty, 0)$, we see that (3.9) is fulfilled. Moreover, if $\alpha<2$ and $-1<\gamma<1-\alpha$, then $\int_{a}^{\infty} G<\infty$ while $\int_{a}^{\infty} H_{\tau}=\infty$, and if $\alpha>2$ and $1-\alpha<\gamma<-1$, then $\int_{a}^{\infty} G=\infty$ while $\int_{a}^{\infty} H_{\tau}<\infty$. Note that in the linear case (i.e., $\left.\alpha=2\right), \lambda^{-\delta-1} G(t) \sim H_{\tau}(t)$ as $t \rightarrow \infty$, and thus half-linear equations exhibit more complex behavior.

Theorem 3.4. Assume that (3.7) holds and $\delta<-1$. Let

$$
\left(r^{1-\beta} \circ \tau\right) \tau^{\prime} \in \mathcal{R} \mathcal{V}(\delta(1-\beta)-\beta) \text { and }\left(\frac{L_{p}(t)}{L_{r}(t)}\right)^{\beta-1} \tau^{\prime}(t) \rightarrow 0
$$

as $t \rightarrow \infty$. Then, $\mathcal{I S} \subset \mathcal{N} \mathcal{R} \mathcal{V}(\varrho)$, where $\varrho$ is defined in $(3.11)$, and $y^{[1]}(t) \in$ $\Pi(t p(t) \Phi(y(\tau(t))))$ for every $y \in \mathcal{I} \mathcal{S}$. In addition, for any $y \in \mathcal{I} \mathcal{S}$ the following hold.

(i) If $\int_{a}^{\infty} H_{\tau}(s) \mathrm{d} s=\infty$, then

$$
y(t)=t^{1-\beta}(t) \exp \left\{\int_{a}^{t}(1+o(1)) \frac{\beta-1}{\Phi(\varrho)} H_{\tau}(s) \mathrm{d} s\right\}
$$

as $t \rightarrow \infty$. Moreover, $\mathcal{S}_{\mathcal{N} \mathcal{R} \mathcal{V}}(\varrho)=\mathcal{S}_{\mathcal{R V}}(\varrho)=\mathcal{I S}=\mathcal{I S}_{\infty, \infty}$.

(ii) If $\int_{a}^{\infty} H_{\tau}(s) \mathrm{d} s<\infty$, then

$$
y(t)=A+\int_{a}^{t} M^{\beta-1} r^{1-\beta}(s) \exp \left\{-\int_{s}^{\infty}(1+o(1)) \frac{\beta-1}{\varrho^{\alpha-1}} H_{\tau}(\xi) \mathrm{d} \xi\right\} \mathrm{d} s
$$


as $t \rightarrow \infty$, where $M=\lim _{t \rightarrow \infty} y^{[1]}(t) \in(0, \infty)$, for some $A \in \mathbb{R}$, and $\mathcal{S}_{\mathcal{N} \mathcal{R} \mathcal{V}}(\varrho)=$ $\mathcal{S}_{\mathcal{R} \mathcal{V}}(\varrho)=\mathcal{I} \mathcal{S}=\mathcal{I S}_{\infty, B}$. Moreover, $M-y^{[1]} \in \mathcal{S} \mathcal{V}$ and

$$
\frac{t^{\delta+\alpha}\left(\tau^{\prime}(t)\right)^{\alpha-1} L_{p}(t)}{\tau^{\delta+\alpha}(t) L_{r}(t)\left(M-y^{[1]}(t)\right)}=o(1)
$$

as $t \rightarrow \infty$.

Remark 3.5. (i) In fact - as a closer examination of the proof shows - because of the first additional condition in (3.12), we could assume only $p \in \mathcal{R} \mathcal{V}(\delta)$ instead of (3.7). Yet, for the proof of regular variation of solutions and asymptotic formulae, we could drop the requirement of $p$ being regularly varying (similarly as $r$ does not need to be regularly varying in Theorem 3.1). But, for simplicity and since we want to express some formulae in terms of $L_{p}, L_{r}$, and thus be in a better correspondence with Theorem 3.1, we prefer to take the stronger assumption.

(ii) In view of Proposition 2.4, the former condition in (3.12) is implied by $\tau^{\prime} \in \mathcal{S} \mathcal{V}$ (provided we assume $r \in \mathcal{R} \mathcal{V}(\delta+\alpha)$ ). The latter condition in (3.12) is implied by (3.9), and $\lim \sup _{t \rightarrow \infty} \tau^{\prime}(t)<\infty$ (provided we assume (3.7)).

(iii) Similarly as in Lemma 3.2 , we can obtain a necessary condition in the setting of Theorem 3.4. In particular, if $\delta<-1, \tau(t) \asymp t$ as $t \rightarrow \infty$, (3.7) is satisfied, and $\mathcal{S}_{\mathcal{N} \mathcal{R} \mathcal{V}}(\varrho) \neq \emptyset$, then (3.9) holds.

In $[13,21]$ (see also [20]), half-linear differential equations with both retarded and advanced arguments are considered. Necessary and sufficient conditions for the existence of a (generalized) $\mathcal{S} \mathcal{V}$ solution and a (generalized) $\mathcal{R} \mathcal{V}(1)$ solution are established. Note that the conditions for the coefficients are more general than ours and take an integral form similar to (3.1) and (3.10). The conditions on the delayed argument are slightly more special than our ones. The methods in [13,21] are based on the results for the associated equation without deviating arguments and the Schauder-Tychonoff fixed point theorem. We emphasize that while in $[13,20,21]$ the existence of $\mathcal{R} \mathcal{V}$ solutions is studied, here we attempt to deal with all positive increasing solutions.

Our results are essentially new also in the linear case, i.e., when $\alpha=2$. The linear version of the existence results mentioned in the previous item can be found in $[9-11]$.

If $\tau(t)=t$, Theorems 3.1 and 3.4 reduce to an improvement of the results in [17, 19]; note that in those papers, conditions (3.7) and (3.9) are assumed throughout.

Next, we establish a generalization of the previous theorems. As we will see, as a by-product, we solve the natural problem arising from Theorem 3.1 and 3.4, namely the missing ("critical") case $\delta=-1$. Also, some equations that do not have $\mathcal{R} \mathcal{V}$ coefficients can be treated by generalized theorems.

We distinguish the following two cases, namely

$$
\int_{a}^{\infty} r^{1-\beta}(s) \mathrm{d} s=\infty
$$

and

$$
\int_{a}^{\infty} r^{1-\beta}(s) \mathrm{d} s<\infty .
$$


In the first case, we denote $R_{D}(t)=\int_{a}^{t} r^{1-\beta}(s) \mathrm{d} s$ and in the second one, we denote $R_{C}(t)=\int_{t}^{\infty} r^{1-\beta}(s) \mathrm{d} s$. Further, $R_{D}^{-1}$ stands for the inverse of $R_{D}$ and $Q^{-1}$ is the inverse of $Q$, where $Q=1 / R_{C}$. It is easy to see that $\left(R_{C} \circ Q^{-1}\right)(s)=1 / s$.

We first give a generalization of Theorem 3.4. Denote

$$
\tau_{D}=R_{D} \circ \tau \circ R_{D}^{-1}, \quad p_{D}=\left(p r^{\beta-1}\right) \circ R_{D}^{-1},
$$

and

$$
q_{D}=\left(\left(\tau_{D}^{\prime} \circ R_{D}\right) R_{D}\right)^{\alpha-1} p .
$$

The following set of conditions will play a role:

$$
\limsup _{t \rightarrow \infty} \frac{t}{\tau_{i}(t)}<\infty, \tau_{i}^{\prime} \in \mathcal{S} \mathcal{V}, \limsup _{t \rightarrow \infty} \tau_{i}^{\prime}(t)<\infty,
$$

where $i=D$ or $i=C$ according to whether $\delta<-1$ or $\delta>-1$, respectively.

Theorem 3.6. Assume that (3.16) holds, $p_{D} \in \mathcal{R V}(-\alpha)$, and $\lim _{t \rightarrow \infty} L_{p_{D}}(t)=$ 0 . Let (1.2) and (3.19) with $i=D$ be fulfilled. Then, for any $y \in \mathcal{I S}$, one has $y \circ R_{D}^{-1} \in \mathcal{N} \mathcal{R} \mathcal{V}(1)$ and the following hold.

(i) If $\int_{a}^{\infty} q_{D}(s) \mathrm{d} s=\infty$, then

$$
y(t)=R_{D}(t) \exp \left\{\int_{a}^{t}(1+o(1))(\beta-1) q_{D 2}(s) \mathrm{d} s\right\}
$$

as $t \rightarrow \infty$ and $y \in \mathcal{I S}_{\infty, \infty}$.

(ii) If $\int_{a}^{\infty} q_{D}(s) \mathrm{d} s<\infty$, then

$$
y(t)=A+\int_{a}^{t} M^{\beta-1} r^{1-\beta}(s) \exp \left\{-\int_{s}^{\infty}(1+o(1))(\beta-1) q_{D}(u) \mathrm{d} u\right\} \mathrm{d} s
$$

as $t \rightarrow \infty$, and $y \in \mathcal{I} \mathcal{S}_{\infty, B}$, with $\lim _{t \rightarrow \infty} y^{[1]}(t)=M \in(0, \infty)$, for some $A \in \mathbb{R}$. In addition, $\left|y^{[1]} \circ R_{D}^{-1}-N\right| \in \mathcal{S} \mathcal{V}$ and

$$
\frac{\left(\tau_{D}^{\prime}\left(R_{D}(t)\right)\right)^{\alpha-1} R_{D}^{\alpha}(t) p(t) r^{\beta-1}(t)}{M-y^{[1]}(t)}=o(1)
$$

as $t \rightarrow \infty$.

The next result is a complement to Theorem 3.6, and generalizes Theorem 3.1. Denote

$$
\tau_{C}=Q \circ \tau \circ Q^{-1}, \quad p_{C}=\left(R_{C}^{2} p r^{\beta-1}\right) \circ Q^{-1}
$$

and

$$
q_{C}=\left(R_{C} p r^{\beta-1}\right)^{\beta-1} .
$$

Theorem 3.7. Assume that (3.17) holds, $p_{C} \in \mathcal{R} \mathcal{V}(\alpha-2)$, and $\lim _{t \rightarrow \infty} L_{p_{C}}(t)=$ 0 . Let (1.2) and (3.19) with $i=C$ be fulfilled. Then, for any $y \in \mathcal{I S}$, one has $y \circ Q^{-1} \in \mathcal{N S} \mathcal{V}$ and the following hold.

(i) If $\int_{a}^{\infty} q_{C}(s) \mathrm{d} s=\infty$, then

$$
y(t)=\exp \left\{\int_{a}^{t}(1+o(1))(\beta-1)^{\beta-1} q_{C}(s) \mathrm{d} s\right\}
$$

as $t \rightarrow \infty$ and $y \in \mathcal{I S}_{\infty, \infty}$. 
(ii) If $\int_{a}^{\infty} q_{C}(s) \mathrm{d} s<\infty$, then

$$
y(t)=N \exp \left\{-\int_{t}^{\infty}(1+o(1))(\beta-1)^{\beta-1} q_{C}(s) \mathrm{d} s\right\}
$$

as $t \rightarrow \infty$ and $y \in \mathcal{I} \mathcal{S}_{B, \infty}$, with $\lim _{t \rightarrow \infty} y(t)=N \in(0, \infty)$. In addition, $\mid y \circ$ $Q^{-1}-N \mid \in \mathcal{S} \mathcal{V}$ and

$$
\frac{R_{C}^{\alpha}(t) p(t) r^{\beta-1}(t)}{\Phi(y(t)-N)}=o(1)
$$

as $t \rightarrow \infty$.

Remark 3.8. In view of Lemma 4.2-(ii), if $\tau$ satisfies (1.2), (1.3), $\tau^{\prime} \in \mathcal{S V}$, $\lim \sup _{t \rightarrow \infty} \tau^{\prime}(t)<\infty$, and $r \in \mathcal{R} \mathcal{V}(\delta+\alpha)$, then (3.19) (which is assumed in Theorems 3.6 and 3.7) is fulfilled, where $i=D$ or $i=C$ according to whether $\delta<-1$ or $\delta>-1$, respectively.

Theorems 3.6 and 3.7 are indeed generalizations of the previous theorems. As easily seen from Lemma 4.2 (see also Remark 3.8), the assumptions of Theorem 3.4 and Theorem 3.1 (supposing here (3.7)) imply the ones of Theorem 3.6 and Theorem 3.7, respectively. Asymptotic formulae in Theorems 3.1 and 3.4 can be obtained from the general ones by applying the Karamata integration theorem (Proposition 2.3) to $R_{D}$ and $R_{C}$.

Theorem 3.6 and Theorem 3.7 allow us to obtain asymptotic formulae also when the coefficients of the equation are not regularly varying. For example, let $p(t)=e^{\gamma t} t^{\omega}, r(t)=e^{\gamma t}$ with $\gamma<0$ and $\omega<0$. Then, (3.16) holds and

$$
p_{D}(s)=\frac{1}{((s+K) \gamma(1-\beta))^{\alpha}} \ln ^{\omega}((s+K) \gamma(1-\beta))^{\frac{1}{\gamma(1-\beta)}}
$$

for some $K \in(0, \infty)$. Therefore, $p_{D} \in \mathcal{R} \mathcal{V}(-\alpha)$ and $L_{p_{D}}(s) \rightarrow 0$ as $s \rightarrow \infty$.

Theorem 3.6 and Theorem 3.7 enable us to cover the missing cases in Theorems 3.1 and 3.4 in the sense of the critical case $\delta=-1$. Let us illustrate this fact when, for simplicity, $r(t)=t^{\alpha-1}$. Assume, in addition, that $p \in \mathcal{R V}(-1)$. Then, (3.7) with $\delta=-1$ holds. It is easy to see that (3.16) is fulfilled. We have $R_{D}(t)=\ln t$ (taking $a=1$ ) and $R_{D}^{-1}(s)=\exp s$. Since $p_{D}(s)=p\left(e^{s}\right)\left(e^{s}\right)^{(\alpha-1)(\beta-1)}=e^{s} p\left(e^{s}\right)=$ $L_{p}\left(e^{s}\right)$, we get $p_{D} \in \mathcal{R} \mathcal{V}(-\alpha)$ provided

$$
L_{p} \circ \exp \in \mathcal{R} \mathcal{V}(-\alpha) .
$$

Under this condition, $L_{p_{D}}(\ln t)=s^{\alpha} p_{D}(s)=s^{\alpha} L_{p}\left(e^{s}\right)=L_{p}(t) \ln ^{\alpha} t\left(\right.$ with $\left.t=e^{s}\right)$. Hence, $\lim _{t \rightarrow \infty} L_{p_{D}}(t)=0$ is implied by

$$
\lim _{t \rightarrow \infty} L_{p}(t) \ln ^{\alpha} t=0 .
$$

Thus, the assumptions of Theorem 3.6 are fulfilled provided (3.24) and (3.25) hold, and we get $y \circ \exp \in \mathcal{N} \mathcal{R} \mathcal{V}(1)$ for $y \in \mathcal{I S}$; in fact, all eventually positive increasing solutions of (1.1) are slowly varying. As for the asymptotic formulae, note that

$$
q_{D}(t)=\left(\frac{t \tau^{\prime}(t)}{\tau(t)}\right)^{\alpha-1} p(t) \ln ^{\alpha-1} t .
$$


Take, for example,

$\omega>0$. Then

$$
p(t)=\frac{1}{t} \cdot \frac{1}{\ln ^{\alpha} t} \cdot \frac{1}{\ln ^{\omega} \ln t}
$$

$$
\left(L_{p} \circ \exp \right)(t)=\frac{1}{t^{\alpha} \ln ^{\omega} t} \in \mathcal{R} \mathcal{V}(-\alpha) .
$$

Assume $t \tau^{\prime}(t) \asymp \tau(t)$ as $t \rightarrow \infty$. Then

$$
q_{D}(t)=\left(\frac{t \tau^{\prime}(t)}{\tau(t)}\right)^{\alpha-1} \frac{\ln ^{-\omega} \ln t}{t \ln t} \asymp \frac{\ln ^{-\omega} \ln t}{t \ln t}
$$

as $t \rightarrow \infty$. Hence, $\int_{a}^{\infty} q_{D}$ diverges [converges] when $\omega \leq 1[\omega>1]$.

Many formulae in the previous two theorems (and their applications) can be expressed in terms of generalized regularly varying functions; this concept was introduced in [7] and has been used in several papers, see, for instance, [21]. Generalized regular variation is defined as follows: A function $f$ is regularly varying of index $\vartheta \in \mathbb{R}$ with respect to $\omega \in C^{1}$, with $\omega^{\prime}>0$ and $\lim _{t \rightarrow \infty} \omega(t)=\infty$, if $f \circ \omega^{-1} \in \mathcal{R} \mathcal{V}(\vartheta)$. Denote the set of all regularly varying functions of index $\vartheta$ with respect to $\omega$ by $\mathcal{R} \mathcal{V}_{\omega}(\vartheta)$. Hence, for example, instead of $y \circ R_{D}^{-1} \in \mathcal{N} \mathcal{R} \mathcal{V}(1)$, we could write $y \in \mathcal{N} \mathcal{R} \mathcal{V}_{R_{D}}(1)$, instead of $p_{D} \in \mathcal{R} \mathcal{V}(-\alpha)$, we could write $p r^{1-\beta} \in$ $\mathcal{R} \mathcal{V}_{R_{D}}(-\alpha)$, and so on.

If $\tau(t)=t$, then Theorem 3.6 (Theorem 3.7) reduces to [18, Theorem 5] ([18, Theorem 6]).

In the last paragraph of this section, we indicate some directions for possible future research.

(i) One of the open problems is to obtain a similar theory for equation (1.1) with the opposite sign condition on the coefficient $p$. In this framework, it would be new even in the ordinary (i.e., non-functional) case.

(ii) A natural problem is to complete our theory also for decreasing solutions. As we could see in the observation following formula (3.10), the difference between the functional and non-functional cases is "bigger" than for increasing solutions. We conjecture that, for example, $\int_{a}^{\infty} r^{1-\beta}(s) \mathrm{d} s=\infty, p \in \mathcal{R} \mathcal{V}(\delta)$ with $\delta<-1$, and (3.10) imply $\mathcal{D S} \subset \mathcal{N S V}$; the assumption about regular variation of $p$ (or some other restriction on $p$ ) cannot be omitted. Once we have guaranteed slow variation of decreasing solutions, asymptotic formulae similar to the ones above can be obtained.

(iii) Another natural problem is examining advanced equations, i.e., the case in which the condition $\tau(t) \leq t$ is replaced by $\tau(t) \geq t$. Here, the situation is "reversed" in the sense that decreasing solutions are easier to be handled than increasing ones. For instance, it is not difficult to show that $\int_{a}^{\infty} r^{1-\beta}(s) \mathrm{d} s=\infty$, $p \in \mathcal{R} \mathcal{V}(\delta)$ with $\delta<-1$, and (3.10) (i.e., the example of the setting is the same as in the previous item) imply $\mathcal{D S} \subset \mathcal{N S} \mathcal{V}$. Such a statement would be a half-linear extension of [5, Theorem 5.1], which deals with linear advanced equations. To the best of the authors' knowledge, this paper is the first one where functional differential equations are analyzed within the framework of regular variation. As for the corresponding result for an advanced equation when (3.1) with $\delta>-1$ holds, nothing is known about slow variation of all increasing solutions even in 
the linear case. On the other hand, practically all arguments which are used to obtain asymptotic formulae, work with appropriate modifications also for advanced equations.

(iv) Since we assume that $L_{p}(t) / L_{r}(t)=t^{\alpha} p(t) / r(t)$ tends to zero as $t \rightarrow \infty$, (i.e., condition (3.9)), it is natural to examine also the condition $\lim _{t \rightarrow \infty} t^{\alpha} p(t) / r(t)$ $=C>0$ or its generalization in the sense of (3.1) or (3.10). In the case of equations without deviating argument, see [18], we can use suitable transformations which lead to a linear second order equation. Then, the results on $\mathcal{S} \mathcal{V}$ solutions can be applied to the transformed equation. A similar method, however, is not known for the associated functional differential equations (even in the linear case).

\section{Proofs of the MAin RESUlts}

In order to prove the main theorems, first we derive several auxiliary statements.

Proof of Proposition 2.4-(ix). (ix) If $f^{\prime} \in \mathcal{R V}(-1)$ and $f(t) \rightarrow \infty$ as $t \rightarrow \infty$, then $f(t)=f(a)+\int_{a}^{t} f^{\prime}(s) \mathrm{d} s \sim \int_{a}^{t} f^{\prime}(s) \mathrm{d} s$. Hence, since $t f^{\prime}(t) \in \mathcal{S} \mathcal{V}$,

$$
t f^{\prime}(t) / f(t) \sim t f^{\prime}(t) / \int_{a}^{t} f^{\prime}(s) \mathrm{d} s \rightarrow 0
$$

as $t \rightarrow \infty$ by Proposition 2.3-(iii). Similarly we proceed when $\left|f^{\prime}\right| \in \mathcal{R} \mathcal{V}(-1)$ and $f(t) \rightarrow 0$ as $t \rightarrow \infty$. As for the case $\vartheta \neq-1$, we use again similar arguments and apply Proposition 2.3-(i) when $\vartheta<-1$, or Proposition 2.3-(ii) when $\vartheta>-1$.

Lemma 4.1. Let $f, g$ be defined on $[a, \infty)$.

(i) If $f \in \mathcal{S} \mathcal{V}$ and $g(t) \asymp t$ as $t \rightarrow \infty$, then $f(g(t)) \sim f(t)$ and $f \circ g \in \mathcal{S V}$.

(ii) If $f \in \mathcal{R} \mathcal{V}(\vartheta), \vartheta \in \mathbb{R}$, and $g(t) \asymp t$ as $t \rightarrow \infty$, then $f(g(t)) \asymp f(t)$.

(iii) If $f \in \mathcal{R} \mathcal{V}(\vartheta), \vartheta \in \mathbb{R}$, and $g(t) \sim t$ as $t \rightarrow \infty$, then $f(g(t)) \sim f(t)$.

Proof. (i) Since $c \leq g(t) / t \leq d, t \in[b, \infty)$, for some $0<c<d<\infty$ and $b \geq a$, in view of the Uniform Convergence Theorem, we have

$$
\frac{f(g(t))}{f(t)}=\frac{f((g(t) / t) t)}{f(t)} \rightarrow 1
$$

as $t \rightarrow \infty$, i.e., $f(g(t)) \sim f(t)$. Therefore, for every $\lambda>0$,

$$
\frac{f(g(\lambda t))}{f(g(t))} \sim \frac{f(\lambda t)}{f(t)} \sim 1
$$

as $t \rightarrow \infty$, i.e., $f \circ g \in \mathcal{S} \mathcal{V}$.

(ii) Let $L(t)=f(t) / t^{\vartheta}$. Then, $L \in \mathcal{S} \mathcal{V}$ and

$$
\frac{f(g(t))}{f(t)}=\left(\frac{g(t)}{t}\right)^{\vartheta} \frac{L(g(t))}{L(t)},
$$

In view of (i), we have

$$
c_{1} \leq c_{2} \frac{L(g(t))}{L(t)} \leq \frac{f(g(t))}{f(t)} \leq d_{2} \frac{L(g(t))}{L(t)} \leq d_{1},
$$

$t \in[b, \infty)$, for some $c_{1}, c_{2}, d_{1}, d_{2} \in(0, \infty)$ and $b \geq a$. 
(iii) As above, let $L(t)=f(t) / t^{\vartheta}$. Then, $L \in \mathcal{S} \mathcal{V}$ and, in view of (4.1) and (i), we have

$$
\frac{f(g(t))}{f(t)} \sim \frac{L(g(t))}{L(t)} \sim 1
$$

as $t \rightarrow \infty$.

Lemma 4.2. Let $\tau_{i}, p_{i}, i=C, D$, be defined as in (3.18) and (3.23).

(i) If (3.7) and (3.9) hold, then $p_{D} \in \mathcal{R} \mathcal{V}(-\alpha)$ and $L_{p_{D}}(s) \rightarrow 0$ as $s \rightarrow \infty$ provided $\delta<-1$, while $p_{C} \in \mathcal{R} \mathcal{V}(\alpha-2)$ and $L_{p_{C}}(s) \rightarrow 0$ as $s \rightarrow \infty$ provided $\delta>-1$.

(ii) Let $\tau$ satisfy (1.2). Then, $\tau_{i}$ fulfills

$$
\tau_{i} \in C^{1}, \tau_{i}^{\prime}>0, \tau_{i}(t) \leq t,
$$

where $i=D$ or $i=C$ according to whether (3.16) or (3.17) holds, respectively. If, in addition, (1.3) holds, $\tau^{\prime} \in \mathcal{S} \mathcal{V}$, $\lim \sup _{t \rightarrow \infty} \tau^{\prime}(t)<\infty$, and $r \in \mathcal{R} \mathcal{V}(\delta+\alpha)$, then (3.19) holds, where $i=D$ or $i=C$ according to whether $\delta<-1$ or $\delta>-1$, respectively.

Proof. (i) The statement follows from Proposition 2.4, the Karamata integration theorem (Proposition 2.3), and the fact that $L_{p}(t) / L_{r}(t) \sim \varrho^{\alpha}\left(L_{p_{D}} \circ R_{D}\right)(t)$ as $t \rightarrow \infty$ provided $\delta<-1$ (the case $\delta>-1$ is similar). The details are left to the reader.

(ii) We will consider only the case $i=D$, since the case $i=C$ is similar. Since $\tau, R_{D}, R_{D}^{-1}$ are $C^{1}$ functions with positive derivatives, $\tau_{D} \in C^{1}$ and $\tau_{D}^{\prime}>0$ follow. Further, from $\tau(t) \leq t$, we have $\tau\left(R_{D}^{-1}(s)\right) \leq R_{D}^{-1}(s)$, and since $R_{D}$ is increasing, we obtain $\tau_{D}(s) \leq s$. From now on, assume that $r \in \mathcal{R} \mathcal{V}(\delta+\alpha)$. Then $R_{D} \in \mathcal{R} \mathcal{V}(\varrho)$ by Proposition 2.4 and the Karamata theorem (Proposition 2.3). If $M>0$ exists such that $t \leq M \tau(t)$, then $R_{D}^{-1}(s) \leq M \tau\left(R_{D}^{-1}(s)\right)$. Consequently, $s \leq R_{D}\left(M \tau\left(R_{D}^{-1}(s)\right)\right) \leq N \tau_{D}(s)$ for some $N>0$, where the existence of $N$ is guaranteed by regular variation of $R_{D}$. Hence, $\lim _{\sup _{t \rightarrow \infty}} t / \tau_{D}(t)<\infty$ follows. Since $\tau^{\prime} \in \mathcal{S} \mathcal{V}$ and $R_{D} \in \mathcal{R} \mathcal{V}(\varrho)$, we have $\tau \in \mathcal{R} \mathcal{V}(1)$ and $R_{D}^{-1} \in \mathcal{R} \mathcal{V}(1 / \varrho)$. Thus, in view of Proposition 2.4,

$$
\begin{aligned}
\tau_{D}^{\prime} & =\left(R_{D}^{\prime} \circ \tau \circ R_{D}^{-1}\right)\left(\tau^{\prime} \circ R^{-1}\right)\left(R^{-1}\right)^{\prime} \\
& \in \mathcal{R} \mathcal{V}\left((\varrho-1) \cdot 1 \cdot \frac{1}{\varrho}+0 \cdot \frac{1}{\varrho}+\left(\frac{1}{\varrho}-1\right)\right)=\mathcal{S} \mathcal{V} .
\end{aligned}
$$

Finally, assume that $\tau^{\prime}(t) \leq M$ for some $M>0$. In view of Lemma 4.1, we have $r^{1-\beta}(\tau(t)) / r^{1-\beta}(t) \asymp 1$ as $t \rightarrow \infty$. Hence,

$$
\tau_{D}^{\prime}(s)=r^{1-\beta}\left(\tau\left(R_{D}^{-1}(s)\right)\right) \tau^{\prime}\left(R_{D}^{-1}(s)\right) \frac{1}{r^{1-\beta}\left(R_{D}^{-1}(s)\right)} \leq N
$$

for some $N>0$.

Lemma 4.3. Let $y$ be a solution of (1.1) and let $\tau$ be differentiable. Then, $u=\operatorname{Cr} \Phi\left(y^{\prime}\right), C \in \mathbb{R}$ satisfies the reciprocal equation

$$
\left(\widetilde{r}(t) \Phi^{-1}\left(u^{\prime}(t)\right)\right)^{\prime}=\widetilde{p}(t) \Phi^{-1}(u(\tau(t))),
$$


where

$$
\widetilde{r}(t)=p^{1-\beta}(t), \quad \widetilde{p}(t)=\tau^{\prime}(t) r^{1-\beta}(\tau(t)) .
$$

If $p \in \mathcal{R} \mathcal{V}(\delta)$ and $\left(r^{1-\beta} \tau^{\prime} \circ \tau\right) \in \mathcal{R} \mathcal{V}(\widetilde{\delta})$ (where the latter condition is implied, e.g., by $r \in \mathcal{R} \mathcal{V}(\delta+\alpha)$ and $\left.\tau^{\prime} \in \mathcal{S} \mathcal{V}\right)$, then $\widetilde{p} \in \mathcal{R} \mathcal{V}(\widetilde{\delta}), \widetilde{r} \in \mathcal{R} \mathcal{V}(\widetilde{\delta}+\beta)$, where $\widetilde{\delta}=\delta(1-\beta)-\beta$. If, moreover, (3.7) holds, then

$$
\frac{L_{\widetilde{p}}(t)}{L_{\widetilde{r}}(t)} \asymp\left(\frac{L_{p}(t)}{L_{r}(t)}\right)^{\beta-1} \tau^{\prime}(t)
$$

as $t \rightarrow \infty$.

Proof. Since $y$ is a solution of (1.1), from the definition of $u$ we have $u^{\prime}(t)=$ $p(t) \Phi(y(\tau))$. Hence, $y(\tau(t))=\Phi^{-1}(1 / p(t)) \Phi^{-1}(u(t))$. Further, $y^{\prime}(t)=\Phi^{-1}(1 / t(t))$ $\Phi^{-1}(u(t))$ and $(y \circ \tau)^{\prime}=\left(y^{\prime} \circ \tau\right) \tau^{\prime}$. Now it is easy to see that $u$ satisfies (4.3). The fact that $\widetilde{p} \in \mathcal{R} \mathcal{V}(\widetilde{\delta})$ and $\widetilde{r} \in \mathcal{R} \mathcal{V}(\widetilde{\delta}+\beta)$ follows from Proposition 2.4. In view of Lemma 4.1, we have

$$
\frac{L_{\widetilde{p}}(t)}{L_{\widetilde{r}}(t)}=\frac{t^{\beta} \tau^{\prime}(t) r^{1-\beta}(\tau(t))}{p^{1-\beta}(t)} \asymp\left(\frac{t^{\alpha} p(t)}{r(t)}\right)^{\beta-1} \tau^{\prime}(t)
$$

as $t \rightarrow \infty$, which implies (4.5).

Lemma 4.4. Put $s=\varphi(t)$ and $x(s)=y\left(\varphi^{-1}(s)\right)$, where $\varphi$ is a differentiable function such that $\varphi^{\prime}(t) \neq 0$, equation (1.1) is transformed into the equation

$$
\frac{\mathrm{d}}{\mathrm{d} s}\left(\widehat{r}(s) \Phi\left(\frac{\mathrm{d} x}{\mathrm{~d} s}(s)\right)\right)=\widehat{p}(s) \Phi(x(\widehat{\tau}(s))),
$$

where

$$
\widehat{p}=\frac{p \circ \varphi^{-1}}{\varphi^{\prime} \circ \varphi^{-1}}, \quad \widehat{r}=\left(r \circ \varphi^{-1}\right) \Phi\left(\varphi^{\prime} \circ \varphi^{-1}\right), \quad \widehat{\tau}=\varphi \circ \tau \circ \varphi^{-1} .
$$

Further,

$$
x^{[1]}(s):=\widehat{r}(s) \Phi\left(\frac{\mathrm{d} x}{\mathrm{~d} s}(s)\right)=y^{[1]}(t) .
$$

If (3.7), (3.16), and $\varphi=R_{D}$ hold, then $\widehat{r}=1$ and $\widehat{p}=\left(p r^{\beta-1}\right) \circ R_{D}^{-1} \in \mathcal{R} \mathcal{V}(-\alpha)$. If (3.7), (3.17), and $\varphi=Q$ hold, then $\widehat{r}(s)=s^{2 \alpha-2}$ and $\widehat{p}=\left(R_{C}^{2} p r^{\beta-1}\right) \circ Q^{-1} \in$ $\mathcal{R} \mathcal{V}(\alpha-2)$.

Proof. The form of the transformed equation follows from the fact that $x(\varphi(t))=$ $y(t)$ and $\frac{\mathrm{d}}{\mathrm{d} t}=\varphi^{\prime}(t) \frac{\mathrm{d}}{\mathrm{d} s}$. The indices of regular variation of $\widetilde{p}$ in both cases can easily be computed via Proposition 2.4 and the Karamata theorem (Proposition 2.3).

Proof of Theorem 3.1. (I) Take $y \in \mathcal{I S}$. Integrating (1.1) from $b$ to $t$, where $b$ is so large that $y(\tau(s))>0$ for $s \geq b$, we get

$$
\begin{aligned}
r(t) \Phi\left(y^{\prime}(t)\right) & =r(b) \Phi\left(y^{\prime}(b)\right)+\int_{b}^{t} p(s) \Phi(y(\tau(s))) \mathrm{d} s \\
& \geq r(b) \Phi\left(y^{\prime}(b)\right)+\Phi(y(\tau(b))) \int_{b}^{t} p(s) \mathrm{d} s \rightarrow \infty
\end{aligned}
$$


as $t \rightarrow \infty$, since $\int_{a}^{\infty} p(s) \mathrm{d} s=\infty$. Thus, $\lim _{t \rightarrow \infty} y^{[1]}(t)=\infty$. The equality in (4.8) and the divergence of the integral lead to the existence of $B>0$ such that

$$
\begin{aligned}
r(t) \Phi\left(y^{\prime}(t)\right) & \leq B \int_{b}^{t} p(s) \Phi(y(\tau(s))) \mathrm{d} s \leq B \Phi(y(\tau(t))) \int_{b}^{t} p(s) \mathrm{d} s \\
& \leq B \Phi(y(t)) \int_{b}^{t} p(s) \mathrm{d} s
\end{aligned}
$$

for large $t$. Hence,

$$
0<\left(\frac{t y^{\prime}(t)}{y(t)}\right)^{\alpha-1} \leq \frac{B t^{\alpha-1}}{r(t)} \int_{b}^{t} p(s) \mathrm{d} s
$$

where the right-hand side tends to zero as $t \rightarrow \infty$ by our assumptions. Therefore, $t y^{\prime}(t) / y(t) \rightarrow 0$ as $t \rightarrow \infty$, and so $y \in \mathcal{N} \mathcal{S} \mathcal{V}$. Note that conditions (1.2), (1.3) imply $\tau \asymp t$. Hence, in view of Lemma 4.1, we have $y(t) \sim y(\tau(t))$ as $t \rightarrow \infty$. Moreover, if $p \in \mathcal{R} \mathcal{V}(\delta)$ with $\delta>-1$, then $p \Phi(y) \in \mathcal{R} \mathcal{V}(\delta)$ by Proposition 2.4. Thus, recalling the equality in (4.8), Proposition 2.3 yields

$$
\begin{aligned}
r(t) \Phi\left(y^{\prime}(t)\right) & \sim \int_{b}^{t} p(s) \Phi(y(\tau(s))) \mathrm{d} s \sim \int_{b}^{t} p(s) \Phi(y(s)) \mathrm{d} s \\
& =\int_{b}^{t} s^{\delta} L_{p}(s) y^{\alpha-1}(s) \mathrm{d} s \\
& \sim \frac{1}{\delta+1} t^{\delta+1} L_{p}(t) y^{\alpha-1}(t)=\frac{1}{\delta+1} t p(t) y^{\alpha-1}(t)
\end{aligned}
$$

as $t \rightarrow \infty$. This implies that $y^{[1]} \in \mathcal{R} \mathcal{V}(\delta+1), \delta+1>0$, therefore $\lim _{t \rightarrow \infty} y^{[1]}(t)=$ $\infty$. Further, from the above asymptotic relation, we have

$$
\frac{y^{\prime}(t)}{y(t)}=(1+o(1)) \Phi^{-1}\left(\frac{t p(t)}{(\delta+1) r(t)}\right)
$$

as $t \rightarrow \infty$.

If $\int_{a}^{\infty} G(s) \mathrm{d} s=\infty$, then we integrate (4.9) from $b$ to $t$ to get

$$
\ln y(t)=\ln y(b)+\int_{b}^{t}(1+o(1)) \frac{G(s)}{\Phi^{-1}(\delta+1)} \mathrm{d} s=\int_{a}^{t}(1+o(1)) \frac{G(s)}{\Phi^{-1}(\delta+1)} \mathrm{d} s,
$$

where the latter equality is true thanks to the divergence of $\int_{a}^{\infty} G(s) \mathrm{d} s$. Indeed, it easily follows that for $\varepsilon(t) \rightarrow 0$ there are $\varepsilon_{1}(t), \varepsilon_{2}(t) \rightarrow 0($ as $t \rightarrow \infty)$ such that $c+\int_{b}^{t}(1+\varepsilon(s)) G(s) \mathrm{d} s=\left(1+\varepsilon_{1}(t)\right) \int_{a}^{t}(1+\varepsilon(s)) G(s) \mathrm{d} s=\int_{a}^{t}\left(1+\varepsilon_{2}(s)\right) G(s) \mathrm{d} s$, where $c$ is constant. Relation (4.10) readily implies formula (3.2). Moreover, we have $\lim _{t \rightarrow \infty} y(t)=\infty$, and so $\mathcal{I S} \subseteq \mathcal{I S} \mathcal{S}_{\infty, \infty}$.

If $\int_{a}^{\infty} G(s) \mathrm{d} s<\infty$, then we integrate (4.9) from $t$ to $\infty$ to get

$$
\ln N-\ln y(t)=\int_{t}^{\infty}(1+o(1)) \frac{G(s)}{\Phi^{-1}(\delta+1)} \mathrm{d} s,
$$

where $N=\lim _{t \rightarrow \infty} y(t)$, and formula (3.3) follows. Clearly, $N$ has to be finite because of the convergence of the integral, and so $\mathcal{I S} \subseteq \mathcal{I S}_{B, \infty}$. 
Now we show that $\mathcal{S} \mathcal{V}$ solutions cannot decrease (when $\delta>-1$ ), i.e., $\mathcal{S}_{\mathcal{S} \mathcal{V}} \subseteq \mathcal{I} \mathcal{S}$. Take $y \in \mathcal{D S}$. Since $y^{[1]}$ is negative increasing, there exists $\lim _{t \rightarrow \infty} y^{[1]}(t)=$ $K \in(-\infty, 0]$. Suppose now that $y \in \mathcal{S} \mathcal{V}$. Then, in view of Lemma 4.1 and Proposition 2.4, py $y^{\alpha-1} \circ \tau \in \mathcal{R} \mathcal{V}(\delta)$. Hence, $\int_{b}^{t} p(s) \Phi(y(\tau(s))) \mathrm{d} s \rightarrow \infty$ as $t \rightarrow \infty$ since $\delta>-1$. This is, however, a contradiction, which can be seen from the equality in (4.8). Since $\mathcal{S}=\mathcal{I S} \cup \mathcal{D S}$, we have proved that $\mathcal{S}_{\mathcal{S V}} \subseteq \mathcal{I} \mathcal{S}$. The other relations between the classes $\mathcal{I} \mathcal{S}, \mathcal{S}_{\mathcal{S} \mathcal{V}}, \mathcal{S}_{\mathcal{N} \mathcal{S} \mathcal{V}}$, and $\mathcal{I} \mathcal{S}_{x, \infty}$ with $x=\infty$ or $x=B$ are clear.

(II) If $p \in \mathcal{R} \mathcal{V}(\delta)$ and $r \in \mathcal{R} \mathcal{V}(\gamma)$, then, because of Proposition 2.3, condition (3.1) reads as

$$
\lim _{t \rightarrow \infty} \frac{t^{\alpha+\delta-\gamma} L_{p}(t)}{L_{r}(t)}=0,
$$

from which we necessarily obtain $\gamma \geq \alpha+\delta$ by Proposition 2.4-(vii). Let $\gamma=\alpha+\delta$

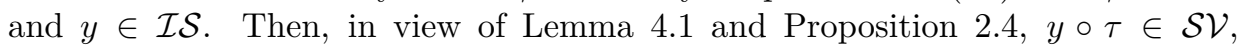
thus $\left(r \Phi\left(y^{\prime}\right)\right)^{\prime}=p \Phi(y \circ \tau) \in \mathcal{R} \mathcal{V}(\delta)$. Since $r(t) \Phi\left(y^{\prime}(t)\right) \sim \int_{t}^{\infty} p(s) y \Phi(\tau(s)) \mathrm{d} s$ as $t \rightarrow \infty$, we have $r \Phi\left(y^{\prime}\right) \in \mathcal{R} \mathcal{V}(\delta+1)$ by Proposition 2.3 , and so $\Phi\left(y^{\prime}\right) \in$ $\mathcal{R} \mathcal{V}(\delta+1-\delta-\alpha)=\mathcal{R} \mathcal{V}(1-\alpha)$. Thus, $y^{\prime} \in \mathcal{R} \mathcal{V}(-1)$ by Proposition 2.4 and $y \in \Pi\left(t y^{\prime}(t)\right)$ by Proposition 2.5. Notice that condition $\gamma>\alpha+\delta$ implies the convergence of $\int_{a}^{\infty} G(s) \mathrm{d} s$. Thus, if $\gamma>\alpha+\delta$ or $\gamma=\alpha+\delta$ and $\int_{a}^{\infty} G(s) \mathrm{d} s<\infty$, as we already know, $\lim _{t \rightarrow \infty} y(t)=N \in(0, \infty)$, and from (4.9),

$$
y^{\prime}(t) \sim \frac{1}{\Phi^{-1}(\delta+1)} G(t) y(t) \sim \frac{N}{\Phi^{-1}(\delta+1)} G(t) \in \mathcal{R} \mathcal{V}((\delta+1-\gamma)(\beta-1))
$$

as $t \rightarrow \infty$. Integrating this relation from $t$ to $\infty$ and using Proposition 2.3, we obtain (3.4). If, in addition, $\gamma=\alpha+\delta$, then, in view of $y \in \Pi$, we get $N-y \in \mathcal{S} \mathcal{V}$ by Proposition 2.5; in our case, this can easily be seen also from (3.4). Formula (3.5) follows from Proposition 2.3-(iii) since $G(t)=\frac{1}{t}\left(\frac{L_{p}(t)}{L_{r}(t)}\right)^{\beta-1}$.

Proof of Lemma 3.2. Take $y \in \mathcal{I S} \cap \mathcal{N S V}$. Set $w=r \Phi\left(y^{\prime} / y\right)$. Then, $w$ is eventually positive and satisfies

$$
w^{\prime}(t)-\frac{\Phi(y(\tau(t)))}{\Phi(y(t))} p(t)+(\alpha-1) r^{1-\beta}(t) w^{\beta}(t)=0
$$

for large $t$. We have

$$
0<\frac{t^{\alpha-1}}{r(t)} w(t)=\left(\frac{t y^{\prime}(y)}{y(t)}\right)^{\alpha-1} \rightarrow 0
$$

as $t \rightarrow \infty$ since $y \in \mathcal{N} \mathcal{S} \mathcal{V}$. Further, $t^{\alpha-1} / r(t)=t^{\alpha-1-\gamma} / L_{r}(t) \in \mathcal{R} \mathcal{V}(\alpha-1-\gamma)$. By our assumptions, $t^{\alpha-1} / r(t) \rightarrow 0$ as $t \rightarrow \infty$. Denote

$$
\Psi(t)=\frac{t^{\alpha-1}}{r(t)} \int_{b}^{t} r^{1-\beta}(s) w^{\beta}(s) \mathrm{d} s,
$$

where $b \geq a$ is such that $y(t)>0$ and $y^{\prime}(t)>0$ for $t \geq b$. If $\int_{b}^{\infty} r^{1-\beta}(s) w^{\beta}(s) \mathrm{d} s<$ $\infty$, then clearly $\Psi(t) \rightarrow 0$ as $t \rightarrow \infty$. Let $\int_{b}^{\infty} r^{1-\beta}(s) w^{\beta}(s) \mathrm{d} s=\infty$. Without loss of generality, we may assume $r \in \mathcal{N} \mathcal{R} \mathcal{V}(\gamma) \cap C^{1}$. Indeed, if $r$ is not normalized or 
is not in $C^{1}$, then we can take $\bar{r} \in \mathcal{N} \mathcal{R} \mathcal{V}(\gamma) \cap C^{1}$ with $\bar{r}(t) \sim r(t)$ as $t \rightarrow \infty$, and we have

$$
\Psi(t) \sim \frac{t^{\alpha-1}}{\bar{r}(t)} \int_{b}^{t} \bar{r}^{1-\beta}(s)\left(\bar{r}(s) \Phi\left(y^{\prime}(s) / y(s)\right)\right)^{\beta} \mathrm{d} s .
$$

The L'Hospital rule yields

$$
\begin{aligned}
\lim _{t \rightarrow \infty} \Psi(t) & =\lim _{t \rightarrow \infty} \frac{r^{1-\beta}(t) w^{\beta}(t)}{r^{\prime}(t) t^{1-\alpha}+(1-\alpha) r(t) t^{-\alpha}} \\
& =\lim _{t \rightarrow \infty} \frac{\left(t y^{\prime}(t) / y(t)\right)^{\alpha}}{t r^{\prime}(t) / r(t)+(1-\alpha)}=\frac{0}{\gamma-\alpha+1}=0 .
\end{aligned}
$$

Integrating (4.11) from $b$ to $t$ and multiplying by $t^{\alpha-1} / r(t)$, we obtain

$$
\frac{t^{\alpha-1}}{r(t)} w(t)-\frac{t^{\alpha-1}}{r(t)} w(b)=\frac{t^{\alpha-1}}{r(t)} \int_{b}^{t} p(s) \Phi\left(\frac{y(\tau(s))}{y(s)}\right) \mathrm{d} s+(\alpha-1) \Psi(t) .
$$

In view of the previous observations, from (4.12) we obtain

$$
\frac{t^{\alpha-1}}{r(t)} \int_{b}^{t} p(s) \Phi\left(\frac{y(\tau(s))}{y(s)}\right) \mathrm{d} s \rightarrow 0
$$

as $t \rightarrow \infty$. Since $y \in \mathcal{S} \mathcal{V}$ and $\tau(t) \asymp t$ as $t \rightarrow \infty$, by Lemma 4.1, there exists $K>0$ such that $y(\tau(t)) / y(t) \geq K$ for $t \geq b$, which, in view of (4.13), implies (3.1). Assuming, in addition, $p \in \mathcal{R} \mathcal{V}(\delta)$, then (3.6) follows by Proposition 2.3.

Proof of Theorem 3.4. For the coefficients $\widetilde{p}, \widetilde{r}$ defined by (4.4), we have that $\widetilde{p} \in \mathcal{R} \mathcal{V}(\widetilde{\delta})$ and $\widetilde{r} \in \mathcal{R} \mathcal{V}(\widetilde{\delta}+\beta)$, where $\widetilde{\delta}:=\delta(1-\beta)-\beta$, and $\lim _{t \rightarrow \infty} L_{\widetilde{p}}(t) / L_{\widetilde{r}}(t)=0$ thanks to Lemma 4.3 and (3.12).

Take $y \in \mathcal{I S}$ and let $u=r \Phi\left(y^{\prime}\right)$. Then, $u \in \mathcal{I S}^{(4.3)}$, recalling the notation (2.1). We have $\widetilde{\delta}>-1$, and thus we can apply Theorem 3.1 to equation (4.3) to obtain $u \in \mathcal{N S \mathcal { V }}$ and $u \in \Pi\left(t u^{\prime}(t)\right)$. Hence, $y^{\prime} \in \mathcal{R} \mathcal{V}((-\delta-\alpha)(\beta-1))$ by Proposition 2.4. Since $\delta<-1$, we have $(-\delta-\alpha)(\beta-1)>-1$, and thus $\int_{b}^{\infty} y^{\prime}(s) \mathrm{d} s=\infty$. Consequently, $y(t) \sim y(t)-y(b)=\int_{b}^{t} y^{\prime}(s) \mathrm{d} s \in \mathcal{N} \mathcal{R} \mathcal{V}(\varrho)$ as $t \rightarrow \infty$, where $\varrho=(-\delta-1) /(\alpha-1)=(-\delta-\alpha)(\beta-1)+1$. Moreover, $y^{[1]} \in \Pi\left(t u^{\prime}(t)\right)=\Pi(t p(t) \Phi(y(\tau(t))))$ and $y \in \mathcal{I S}_{\infty}$ since the index $\varrho$ of regular variation is positive. We have shown that $\mathcal{I S} \subset \mathcal{N} \mathcal{R} \mathcal{V}(\varrho)$. Next, we derive asymptotic formulae by applying again Theorem 3.1.

If $\int^{\infty}(s \widetilde{p}(s) \widetilde{r}(s))^{\alpha-1} \mathrm{~d} s=\infty$, then

$$
u(t)=\exp \left\{\int_{a}^{t}(1+o(1)) \Phi\left(\frac{s \widetilde{p}(s)}{(\widetilde{\delta}+1) \widetilde{r}(s)}\right) \mathrm{d} s\right\}
$$

as $t \rightarrow \infty$ and $u \in \mathcal{I S}_{\infty}^{(4.3)}$.

If $\int^{\infty}(s \widetilde{p}(s) \widetilde{r}(s))^{\alpha-1} \mathrm{~d} s<\infty$, then

$$
u(t)=M \exp \left\{-\int_{t}^{\infty}(1+o(1)) \Phi\left(\frac{s \widetilde{p}(s)}{(\widetilde{\delta}+1) \widetilde{r}(s)}\right) \mathrm{d} s\right\}
$$

as $t \rightarrow \infty$ and $u \in \mathcal{I S}_{B}^{(4.3)}$, where $M=\lim _{t \rightarrow \infty} u(t) \in(0, \infty)$. 
Since

$$
\left(\frac{t \widetilde{p}(t)}{\widetilde{r}(t)}\right)^{\alpha-1}=H_{\tau}(t) \text { and } \widetilde{\delta}+1=\varrho,
$$

from (4.14) and $y^{\prime}(t) \sim \varrho y(t) / t$, under the assumption $\int_{a}^{\infty} H_{\tau}=\infty$, we get

$$
y(t)=(1+o(1)) \frac{1}{\varrho} \operatorname{tr}^{1-\beta}(t) \exp \left\{\int_{a}^{t}(1+o(1)) \frac{\beta-1}{\varrho^{\alpha-1}} H_{\tau}(s) \mathrm{d} s\right\}
$$

as $t \rightarrow \infty$. Because of the divergence of $\int_{a}^{\infty} H_{\tau}$, the expression $(1+o(1)) / \varrho$ can be included in the $(1+o(1))$ term inside the integral in (4.16) (similarly as in (4.10)), and thus we obtain formula (3.13). Since $y \in \mathcal{I} \mathcal{S}_{\infty}$ and $u \in \mathcal{I S}_{\infty}^{(4.3)}$, we have $y \in \mathcal{I S}_{\infty, \infty}$.

Now assume that $\int_{a}^{\infty} H_{\tau}<\infty$. Then, (4.15) implies

$$
y^{\prime}(t)=r^{1-\beta}(t) M^{\beta-1} \exp \left\{-\int_{t}^{\infty}(1+o(1)) \frac{\beta-1}{\varrho^{\alpha-1}} H_{\tau}(s) \mathrm{d} s\right\}
$$

as $t \rightarrow \infty$. Integrating from $b$ to $t$, replacing the lower limit in the integral by $a$ and $y(b)$ by a suitable $A$, we obtain formula (3.14). Moreover, $y \in \mathcal{I S}_{\infty, B}$, in view of $y \in \mathcal{I} \mathcal{S}_{\infty}$ and $u \in \mathcal{I S}_{B}^{(4.3)}$. From Theorem 3.1 we know that

$$
|M-u| \in \mathcal{S} \mathcal{V} \text { and } \frac{L_{\widetilde{p}}^{\alpha-1}(t)}{L_{\widetilde{r}}^{\alpha-1}(M-u(t))}=o(1)
$$

as $t \rightarrow \infty$. Noting that, by Lemma 4.1,

$$
\frac{L_{\widetilde{p}}^{\alpha-1}(t)}{L_{\widetilde{r}}^{\alpha-1}} \sim\left(\frac{t}{\tau(t)}\right)^{\delta+\alpha}\left(\tau^{\prime}(t)\right)^{\alpha-1} \frac{L_{p}(t)}{L_{r}(t)}
$$

as $t \rightarrow \infty$, we get $\left|M-y^{[1]}\right| \in \mathcal{S} \mathcal{V}$ and (3.15) easily follow. The equalities among the subclasses follow from the relations $\mathcal{I S} \subseteq \mathcal{S}_{\mathcal{N} \mathcal{R} \mathcal{V}}(\varrho) \subseteq \mathcal{S}_{\mathcal{R V}}(\varrho) \subseteq \mathcal{I S} \subseteq \mathcal{I S}_{\infty, x} \subseteq$ $\mathcal{I S}, x=B$ or $x=\infty$ according to whether $\int_{a}^{\infty} H_{\tau}$ converges or diverges, respectively; note that a regularly varying solution of (1.1) with a positive index is necessarily increasing.

Proof of Theorem 3.6. First note that, in view of Lemma 4.2-(ii), (1.2) implies (4.2) with $i=D$. Take $y \in \mathcal{I S}$. Then, $x(s)=y(t)$, with $s=R_{D}(t)$, satisfies equation (4.6), where $\widehat{r}=1, \widehat{p}=p_{D}, \widehat{\tau}=\tau_{D}$, and $x \in \mathcal{I S}^{(4.6)}$, see Lemma 4.4. Note that the interval $[a, \infty)$ is transformed into $[b, \infty)$ for some $b$. Since $\widehat{r} \in \mathcal{S} \mathcal{V}$ and $\widehat{p} \in \mathcal{R} \mathcal{V}(-\alpha)$, we can apply Theorem 3.4 to equation (4.6); in fact, here $\delta=-\alpha<-1$. We get $x \in \mathcal{N} \mathcal{R} \mathcal{V}(1)$ since $\delta=-\alpha$ implies $\varrho=1$. Moreover,

$$
x(s)=s \exp \left\{\int_{b}^{s}(1+o(1))(\beta-1)\left(u \widehat{\tau}^{\prime}(u)\right)^{\alpha-1} \widehat{p}(u) \mathrm{d} u\right\}
$$

as $s \rightarrow \infty$, with $x \in \mathcal{I S}_{\infty, \infty}^{(4.6)}$ provided $\int_{b}^{\infty}\left(u \widehat{\tau}^{\prime}(u)\right)^{\alpha-1} \widehat{p}(u) \mathrm{d} u=\infty$, while

$$
x(s)=B+\int_{b}^{s} M^{\beta-1} \exp \left\{-\int_{\xi}^{\infty}(1+o(1))(\beta-1)\left(u \widehat{\tau}^{\prime}(u)\right)^{\alpha-1} \widehat{p}(u) \mathrm{d} u\right\} \mathrm{d} \xi
$$


as $s \rightarrow \infty$, for some $B \in \mathbb{R}$, with $x \in \mathcal{I S}_{\infty, B}^{(4.6)}$ provided $\int_{b}^{\infty}\left(u \widehat{\tau}^{\prime}(u)\right)^{\alpha-1} \widehat{p}(u) \mathrm{d} u=\infty$. Since $\widehat{p}=p_{D}$ and $\widehat{\tau}=\tau_{D}$, substitutions $v=R_{D}^{-1}(u)$ and $\eta=R_{D}^{-1}(\xi)$ in the integrals (4.17) and (4.18) yield formulae (3.20) and (3.21). The fact that $y \in$ $\mathcal{I S}_{\infty, i}, i \in\{B, \infty\}$, follows from $x \in \mathcal{I S}_{\infty, i}^{(4.6)}$ and $(4.7)$, recalling that $x\left(R_{D}^{-1}(s)\right)=$ $y(t), \widehat{p}=p_{D}$, and $\widehat{\tau}=\tau_{D}$. From $\left|M-x^{[1]}\right| \in \mathcal{S} \mathcal{V}$, we obtain $\left|M-y^{[1]} \circ R_{D}^{-1}\right| \in \mathcal{S} \mathcal{V}$. In view of Lemma 4.2 and condition (3.19), we have $\tau_{D}(s) \asymp s$ as $s \rightarrow \infty$. Hence, expressing relation (3.15) in terms of our setting, we obtain

$$
\frac{\left(\widehat{\tau}^{\prime}(s)\right)^{\alpha-1} L_{\widehat{p}}(s)}{M-x^{[1]}(s)}=o(1)
$$

as $s \rightarrow \infty$. Recalling that $L_{\widehat{p}}(s)=s^{\alpha} \widehat{p}(s)=R_{D}^{\alpha}(t) p(t) r^{\beta-1}(t)$, we get (3.22).

Proof of Theorem 3.7. The proof is similar to that of Theorem 3.6. Here, for $y \in \mathcal{S}$, we make the substitution $x(s)=y(t)$ with $s=Q(t)$. In view of Lemma 4.4, $x$ satisfies equation (4.6), where $\widehat{r}=s^{2 \alpha-2} \in \mathcal{R} \mathcal{V}(2 \alpha-2), \widehat{p}=p_{C} \in \mathcal{R} \mathcal{V}(\alpha-2)$, and $\widehat{\tau}=\tau_{C}$. From Lemma 4.2-(ii), condition (4.2) with $i=C$ holds. Since, in fact, $\delta=\alpha-2>-1$, we can apply Theorem 3.1 to equation (4.6), where we consider $x \in \mathcal{I S}^{(4.6)}$ (i.e., $y \in \mathcal{I S}$ ). The details are left to the reader.

\section{REFERENCES}

[1] N. H. Bingham, C. M. Goldie and J. L. Teugels, Regular Variation, Encyclopedia of Mathematics and its Applications, Vol. 27, Cambridge University Press, 1987.

[2] O. Došlý and P. Řehák, Half-linear Differential Equations, Elsevier, North Holland, 2005.

[3] J.L. Geluk, On slowly varying solutions of the linear second order differential equation, Publications de l'Institut Mathématique, Nouvelle Série 48 (1990), 52-60.

[4] J. L. Geluk and L. de Haan, Regular Variation, Extensions and Tauberian Theorems, CWI Tract 40, Amsterdam, 1987.

[5] L. J. Grimm and L. M. Hall, Self adjoint differential equations and Karamata functions, in: D. Bainov et al. (eds.), Proceedings of the Third International Colloquium on Differential Equations, Plovdiv, Bulgaria, 1992, Utrecht, 1993, pp. 77-87.

[6] L. de Haan, On Regular Variation and its Applications to the Weak Convergence of Sample Extremes, Mathematisch Centrum Amsterdam, 1970.

[7] J. Jaroš and T. Kusano, Self-adjoint differential equations and generalized Karamata functions, Bulletin, Classe des Sciences Mathématiques et Naturelles, Sciences Mathématiques 29 (2004), 25-60.

[8] J. Jaroš, T. Kusano and T. Tanigawa, Nonoscillation theory for second order half-linear differential equations in the framework of regular variation, Results in Mathematics 43 (2003), 129-149.

[9] T. Kusano and V. Marić, On a class of functional differential equations having slowly varying solutions, Publications de l'Institut Mathématique, Nouvelle Série 80 (2006), 207217.

[10] T. Kusano and V. Marić, Slowly varying solutions of functional differential equations with retarded and advanced arguments, Georgian Mathematical Journal 14 (2007), 301-314.

[11] T. Kusano and V. Marić, Regularly varying solutions to functional differential equations with deviating argument, Bulletin, Classe des Sciences Mathématiques et Naturelles, Sciences Mathématiques 32 (2007), 105-128.

[12] T. Kusano and B. S. Lalli, On oscillation of half-linear functional-differential equations with deviating arguments, Hiroshima Mathematical Journal 24 (1994), 549-563.

[13] J. Manojlović and T. Tanigawa, Regularly varying solutions of half-linear differential equations with retarded and advanced arguments, Mathematica Slovaca 65 (2015), 1361-1402. 
[14] V. Marić, Regular Variation and Differential Equations, Lecture Notes in Mathematics 1726, Springer-Verlag, Berlin-Heidelberg-New York, 2000.

[15] F. Neuman, Transformation and canonical forms of functional-differential equations, Proceedings of the Royal Society of Edinburgh, Section A, Mathematics 115 (1990), 349-357.

[16] M. Pituk, Oscillation of a linear delay differential equation with slowly varying coefficient, Applied Mathematics Letters 73 (2017), 29-36.

[17] P. Řehák, Methods in half-linear asymptotic theory, Electronic Journal of Differential Equations 2016 (2016), Paper No. 267, 27 pp.

[18] P. Řehák, Asymptotic formulae for solutions of half-linear differential equations, Applied Mathematics and Computation 292 (2017), 165-177.

[19] P. Řehák and V. Taddei, Solutions of half-linear differential equations in the classes Gamma and $P i$, Differential and Integral Equations 29 (2016), 683-714.

[20] T. Tanigawa, Regularly varying solutions of half-linear functional differential equations with retarded arguments, Acta Mathematica Hungarica 120 (2008), 53-78.

[21] T. Tanigawa, Generalized regularly varying solutions of second order nonlinear differential equations with deviating arguments, Memoirs on Differential Equations and Mathematical Physics 57 (2012), 123-162.

Serena Matucci, Department of Mathematics and Informatics "U. Dini", University of Florence, I-50139 Florence, Italy

e-mail: serena.matucci@unifi.it

Pavel Řehák, Institute of Mathematics, FME, Brno University of Technology, Technická 2, Brno, CZ-61669, Czech Republic

e-mail: rehak.pavel@fme.vutbr.cz 